\title{
On Uniqueness of Strong Solution of Stochastic Systems
}

\author{
Gang Li and Ming Chen \\ School of Mathematics and System Science, Shandong University of Science and Technology, Qingdao 265590, China \\ Correspondence should be addressed to Gang Li; ligangccm@163.com
}

Received 25 October 2013; Revised 5 January 2014; Accepted 11 January 2014; Published 24 February 2014

Academic Editor: Antonio Suárez

Copyright (C) 2014 G. Li and M. Chen. This is an open access article distributed under the Creative Commons Attribution License, which permits unrestricted use, distribution, and reproduction in any medium, provided the original work is properly cited.

\begin{abstract}
A kind of the well-known matrix Riccati equations which arise in certain stochastic optimal problems is investigated. With the aid of the operator spectrum and the generalized Lyapunov equation approach, we give a sufficient condition for existence and uniqueness of the strong solution related to the critical mean square stabilization of stochastic linear controlled systems, which proves Conjecture 10 in (Zhang et al. (2008)) to a large extent. In addition, we get some properties of the strong solution. At last, we give a kind of stochastic system which has only a strong solution by an example.
\end{abstract}

\section{Introduction}

As is well known, a great deal of attention has recently been given to systems with stochastic multiplicative noise, due to the fact that the modelling of uncertainties using this kind of formulation has been found of many applications in engineering, finance, and so on. With the appearing of two classic books $[1,2]$, stochastic stability and stabilization of Itô differential systems were investigated by many researchers for several decades; we refer the readers to [3-5] and the references therein. More specifically, for linear time-invariant stochastic (LTIS) systems, most works are concentrated on the investigation of mean square stabilization, which has important applications in system analysis and design. Some necessary and sufficient conditions for the mean square stabilization of LTIS systems were obtained in terms of the generalized algebraic Riccati equation (GARE) in [6], the linear matrix inequality (LMI) in [7], or the spectra of some operators in $[4,8]$. In addition, as well known in the linear system theory, complete observability and detectability play important roles in system analysis and design. Complete observability was extended to define exact observability" of stochastic Itô systems in [4]. Some of the other works on this subject can be found, for instance, [8-11].

In this paper, we concentrate our attention on the strong solution of GARE related to thecritical mean square stabiliza- tion of stochastic systems. The main novelty is to analyze the systems with stochastic multiplicative noise in both state and control and to give the condition of uniqueness of the strong solution of GARE. In Section 2, we give some preliminaries including some definitions and theorems we will use. In Section 3, we mainly study the condition of uniqueness of the strong solution of a kind of stochastic systems and give a positive proof of Conjecture 10 in [9]. In Section 4, we give an example where the stochastic system has only a strong solution.

For convenience, we adopt the following traditional notations: $S^{n}$ : the set of all symmetric matrices, their components may be complex; $R^{k}$ : the $k$-dimensional real vector space with the usual inner product $\langle\cdot, \cdot\rangle$ and the corresponding 2-norm $\|\cdot\| ; R^{m \times n}$ : the space of all $m \times n$ matrices with entries in $R ; A^{\prime}(\operatorname{Ker}(A))$ : the transpose (kernel space) of a matrix $A ; A \geq 0(A>0)$ : the positive semidefinite (positive definite) symmetric matrix $A$; $I$ : the identity matrix; $\sigma(L)$ : the spectral set of the operator or matrix $L ; C^{-}\left(C^{-0}\right)$ : the open left (closed left) hand side complex plane; $L_{F_{t}}^{2}\left(R^{+}, R^{n}\right)$ : the space of nonanticipative stochastic processes $x(t) \in R^{n}$ with respect to an increasing $\sigma$-algebra $\left\{F_{t}\right\}_{t \geq 0}$ satisfying $E \int_{0}^{\infty}|x(t)|^{2} d t<$ $\infty ; R^{+}$: the set of all positive real numbers. Finally, we make the assumption throughout this paper that all systems have real coefficients. 


\section{Preliminaries}

In order to illustrate our main results in the next Section 3, first of all, we give some definitions and theorems we will use. Consider the following stochastic Itô differential system:

$$
\begin{gathered}
d x=A x d t+C x d w, \\
x(0)=x_{0} \in R^{n},
\end{gathered}
$$

where $A \in R^{n \times n}$ and $C \in R^{n \times n}$ are real constant matrices and $w(\cdot)$ is a standard one-dimensional Wiener process defined on the filtered probability space $\left(\Omega, F, P, F_{t}\right)$ with $F_{t}=$ $\sigma\{w(s) \mid 0 \leq s \leq t\}$. System (1) or $(A, C)$ is called mean square stable, if $\lim _{t \rightarrow+\infty} E\|x(t)\|^{2}=0$ for any deterministic initial state $x(0) \in R^{n}$. If we set $X(t)=E x(t) x^{\prime}(t)$, by Itô's formula, $X(t)$ satisfies the following generalized Lyapunov differential equation:

$$
\begin{gathered}
\dot{X}=A X+X A^{\prime}+C X C^{\prime}, \\
X(0)=x(0) x^{\prime}(0) .
\end{gathered}
$$

Here $\dot{X}$ denotes the time-derivative of $X$. Motivated by (2), we introduce the following linear Lyapunov operator:

$$
\mathscr{L}_{A, C}: X \in S^{n} \longmapsto A X+X A^{\prime}+C X C^{\prime} \in S^{n} .
$$

In [10], the following theorem gives a necessary and sufficient condition for the mean square stability of system (1) via the spectrum of $\mathscr{L}_{A, C}$, which is called "spectral criterion." Other spectral criteria were found in $[6,12]$.

Theorem 1. System (1) is asymptotical mean square stable if and only if $\sigma\left(\mathscr{L}_{A, C}\right) \subset C^{-}$.

For a state feedback control law $u=K x$, one introduces a linear operator $\mathscr{L}_{K}$ associated with the closed-loop system:

$$
\begin{gathered}
d x=(A+B K) x d t+(C+D K) x d w \\
x(0)=x_{0} \in R^{n}
\end{gathered}
$$

which is defined as $\mathscr{L}_{K}: X \in S^{n} \rightarrow(A+B K) X+$ $X(A+B K)^{\prime}+(C+D K) X(C+D K)^{\prime}$. Where $A \in R^{n \times n}, B \in$ $R^{n \times m}, C \in R^{n \times n}, D \in R^{n \times m}$, and $K \in R^{m \times n}$ are all real constant matrices.

Definition 2. System (4) (or $(A, B, C, D)$ ) is mean square stabilizable if and only if $\sigma\left(\mathscr{L}_{K}\right) \subset C^{-}$for some $K \in R^{m \times n}$.

Definition 3. System $(A, B, C, D)$ is critical mean square stabilizable if and only if $\mathscr{L}_{K} \subset C^{-0}$ for some $K \in R^{m \times n}$.

The following theorems give the relations between the Lyapunov operator spectrum of deterministic system and the Lyapunov operator spectrum of stochastic system (see [9]), which will be used in the proof of the main results.

Theorem 4. For system (1), if $\sigma\left(\mathscr{L}_{A, C}\right) \subset C^{-0}$, then $\sigma(A) \subset$ $C^{-0}$.
Theorem 5. For system (1), if $\sigma\left(\mathscr{L}_{A, C}\right) \subset C^{-}$, then $\sigma(A) \subset C^{-}$. In infinite horizon linear quadratic optimal control, stochastic stability and filtering (see, e.g. [3, 13, 14]), the following general algebra Ricatti equation

$$
\begin{array}{r}
P A+A^{\prime} P+C^{\prime} P C+Q-\left(P B+C^{\prime} P D\right) \\
\times\left(R+D^{\prime} P D\right)^{-1}\left(B^{\prime} P+D^{\prime} P C\right)=0, \\
R+D^{\prime} P D>0, \quad Q \geq 0, \quad R>0
\end{array}
$$

has many applications. In fact, GARE (5) is a generalized version of the following deterministic algebraic Riccati equation (DARE):

$$
\begin{array}{r}
P A+A^{\prime} P+Q-P B R^{-1} B^{\prime} P=0, \\
R>0, \quad Q \geq 0 .
\end{array}
$$

In [9], the following definitions and theorem are given.

Definition 6. A solution $P \in S^{n}$ of GARE (5) is called a feedback stabilizing solution if $\sigma\left(\mathscr{L}_{K}\right) \subset C^{-} ; P$ is called a strong solution if $\sigma\left(\mathscr{L}_{K}\right) \subset C^{-0}$, where $K=-(R+$ $\left.D^{\prime} P D\right)^{-1}\left(B^{\prime} P+D^{\prime} P C\right)$.

Definition 7. A solution $\widehat{P} \in S^{n}$ of GARE (5) is called the maximal solution if $\widehat{P}-P \geq 0$ for any solution $P \in S^{n}$ of GARE (5).

Theorem 8. Suppose system $(A, B, C, D)$ is mean square stabilizable, the weighting real matrices $Q \geq 0, R>0$. Let $\widehat{P}$ be any real symmetric solution of the GARE

$$
\begin{array}{r}
P A+A^{\prime} P+C^{\prime} P C+\widehat{Q}-\left(P B+C^{\prime} P D\right) \\
\times\left(\widehat{R}+D^{\prime} P D\right)^{-1}\left(B^{\prime} P+D^{\prime} P C\right)=0, \\
\widehat{R}+D^{\prime} P D>0 .
\end{array}
$$

If $R \geq \widehat{R}$ and $Q \geq \widehat{Q}$, then GARE (5) has maximal solution $P \geq 0$ and $P \geq \widehat{P}$. Moreover, $P$ is a strong solution.

Remark 9. We know that if system $(A, B, C, D)$ is mean square stabilizable, then the maximal solution of (5) is a feedback stabilizing solution, but a strong solution of (5) may not be a feedback stabilizing solution.

In the following section, we will study the uniqueness of the strong solution of a kind of stochastic system; namely, the strong solution of (5) must be the maximal solution. Accordingly, we give a positive proof of Conjecture 10 in [9]. To end this section, we give this conjecture.

Conjecture 10. Assume system $(A, B, C, D)$ is mean square stabilizable, if GARE (5) admits a strong solution $P \in S^{n}$, then $P$ is also the maximal solution.

\section{Main Results}

In this section, we will give the condition of uniqueness of the strong solution of GARE (5). Here we only investigate the 
strong solution of GARE (5) related to a class of stochastic systems.

Consider the following stochastic system:

$$
\begin{gathered}
d x=(A x+B u) d t+C x d w, \\
x(0)=x_{0} \in R^{n}
\end{gathered}
$$

GARE (5) may become

$$
\begin{array}{r}
P A+A^{\prime} P+C^{\prime} P C+Q-P B R^{-1} B^{\prime} P=0, \\
Q \geq 0, \quad R>0 .
\end{array}
$$

Now we present a very useful lemma which helps us give the condition of uniqueness of the strong solution.

Lemma 11. Let $P_{1} \in S^{n}$ be a solution of GARE (9). Then $P_{2} \in$ $S^{n}$ satisfies GARE (9) if and only if $D_{0}=P_{1}-P_{2}$ satisfies $A_{0}^{\prime} D_{0}+$ $D_{0} A_{0}+C^{\prime} D_{0} C+D_{0} B R^{-1} B^{\prime} D_{0}=0$, where $A_{0}=A-B R^{-1} B^{\prime} P_{1}$.

Proof. We know that the solutions $P_{1}$ and $P_{2}$ of GARE (9) satisfy the following two equations:

$$
\begin{array}{r}
P_{1} A+A^{\prime} P_{1}+C^{\prime} P_{1} C+Q-P_{1} B R^{-1} B^{\prime} P_{1}=0, \\
Q \geq 0, \quad R>0, \\
P_{2} A+A^{\prime} P_{2}+C^{\prime} P_{2} C+Q-P_{2} B R^{-1} B^{\prime} P_{2}=0, \\
Q \geq 0, \quad R>0 .
\end{array}
$$

Two above equations are subtracted, by a series of computations, and we have

$$
A_{0}^{\prime} D_{0}+D_{0} A_{0}+C^{\prime} D_{0} C+D_{0} B R^{-1} B^{\prime} D_{0}=0
$$

The proof of Lemma 11 is complete.

Lemma 12. Suppose system (8) (or $(A, B, C))$ is mean square stabilizable, and the weighting real matrices $Q \geq 0, R>0$. Then GARE (9) has the maximal solution which is only a feedback stabilizing solution of system (8).

Proof. The proof is easy, and we omit it.

Theorem 13. Assume that system $(A, B, C)$ is mean square stabilizable and $\widetilde{P}$ denotes the maximal solution of GARE (9). There does not exist the other strong solution of GARE (9) when $\left|\operatorname{Re}\left(\lambda_{i}\right)\right|>\left|\operatorname{Re}\left(\mu_{i} \mu_{j}\right)\right|$ for $\lambda_{i} \in \sigma\left(A-B R^{-1} B^{\prime} \widetilde{P}\right)$ and $\mu_{i}, \mu_{j} \in \sigma(C)$; that is, there is only a strong solution of GARE (9).

Proof. By Theorems 10 and 12 in [3], if system $(A, B, C)$ is mean square stabilizable, then GARE (9) has the maximal solution $\widetilde{P}$. By Theorem 8 , it is also a strong solution.
By contradiction, now assume that $\widehat{P}$ is the other strong solution of GARE (9). Then

$$
\begin{array}{r}
\widetilde{P} A+A^{\prime} \widetilde{P}+C^{\prime} \widetilde{P} C+Q-\widetilde{P} B R^{-1} B^{\prime} \widetilde{P}=0, \\
Q \geq 0, \quad R>0, \\
\widehat{P} A+A^{\prime} \widehat{P}+C^{\prime} \widehat{P} C+Q-\widehat{P} B R^{-1} B^{\prime} \widehat{P}=0, \\
Q \geq 0, \quad R>0 .
\end{array}
$$

In what follows, we will prove that $\widetilde{P}=\widehat{P}$. Let $H=\widetilde{P}-\widehat{P}(\geq 0)$. If $H>0$, from Lemma 11, subtracting (13) from (12), we have

$$
A_{0}^{\prime} H+H A_{0}+C^{\prime} H C+H B R^{-1} B^{\prime} H=0 ;
$$

here $A_{0}=A-B R^{-1} B^{\prime} \widetilde{P}$. From (14), we have

$$
A_{0}+B R^{-1} B^{\prime} H=-H^{-1} A_{0}^{\prime} H-H^{-1} C^{\prime} H C .
$$

Since $\left|\operatorname{Re}\left(\lambda_{i}\right)\right|>\left|\operatorname{Re}\left(\mu_{i} \mu_{j}\right)\right|$ for $\lambda_{i} \in \sigma\left(A_{0}\right)$ and $\mu_{i}, \mu_{j} \epsilon$ $\sigma(C)$, there exists a $\theta_{i} \in \sigma\left(-H^{-1} A_{0}^{\prime} H-H^{-1} C^{\prime} H C\right)$ such that $\operatorname{Re}\left(\theta_{i}\right)>0 . \sigma\left(-H^{-1} A_{0}^{\prime} H-H^{-1} C^{\prime} H C\right) \subset \sigma\left(A_{0}+B R^{-1} B^{\prime} H\right)=$ $\sigma\left(A_{0}+B R^{-1} B^{\prime}(\widetilde{P}-\widehat{P})\right)=\sigma\left(A-B R^{-1} B^{\prime} \widetilde{P}+B R^{-1} B^{\prime}(\widetilde{P}-\widehat{P})\right)=$ $\sigma\left(A-B R^{-1} B^{\prime} \widehat{P}\right) \subset C^{-0}$, which is a contradiction. So $H>0$ does not hold. Hence $H \geq 0$. If $H \geq 0$ and $H \neq 0$; there exists $x \in R^{n}$ such that $H x=0$. Premultiplying by $x^{\prime}$ and postmultiplying by $x$ in (14) yield

$$
x^{\prime} A_{0}^{\prime} H x+x^{\prime} H A_{0} x+x^{\prime} C^{\prime} H C x+x^{\prime} H B R^{-1} B^{\prime} H x=0 ;
$$

we see that $H C x=0$. Postmultiplying by $x$ in (14) yields

$$
A_{0}^{\prime} H x+H A_{0} x+C^{\prime} H C x+H B R^{-1} B^{\prime} H x=0,
$$

we see that $H A_{0} x=0$. Hence $\operatorname{Ker}(H)$ is an invariant subspace with respect to both $A_{0}$ and $C$. By matrix theory, there exists an orthogonal matrix $S$ such that

$$
\begin{array}{cc}
S^{\prime} A_{0} S=\left(\begin{array}{cc}
A_{1} & * \\
0 & A_{2}
\end{array}\right), & S^{\prime} C S=\left(\begin{array}{cc}
C_{1} & * \\
0 & C_{2}
\end{array}\right), \\
S^{\prime} H S=\left(\begin{array}{cc}
0 & 0 \\
0 & W
\end{array}\right), & S^{\prime} B S=\left(\begin{array}{l}
B_{1} \\
B_{2}
\end{array}\right) .
\end{array}
$$

And premultiplying by $S^{\prime}$ and postmultiplying by $S$ in (14) yield

$$
\begin{gathered}
S^{\prime} A_{0}^{\prime} S S^{\prime} H S+S^{\prime} H S S^{\prime} A_{0} S+S^{\prime} C^{\prime} H S S^{\prime} C S \\
+S^{\prime} H S S^{\prime} B S S^{\prime} R^{-1} S S^{\prime} B^{\prime} S S^{\prime} H S=0
\end{gathered}
$$

So

$$
A_{2}^{\prime} W+W A_{2}+C_{2}^{\prime} W C_{2}+M=0, \quad M \geq 0 .
$$

Here,

$$
S^{\prime} H B R^{-1} B^{\prime} H S=\left(\begin{array}{cc}
0 & * \\
0 & M
\end{array}\right) .
$$


In turn, $W>0$ in (20) satisfies

$$
A_{2}^{\prime} W+W A_{2}+C_{2}^{\prime} W C_{2} \leq 0 .
$$

Hence $\sigma\left(\mathscr{L}_{A_{2}, C_{2}}\right) \subset C^{-0}$ follows from Lyapunov theory and whenever $\operatorname{Re}\left(\lambda_{i}^{2}\right)=0, \lambda_{i} \in \sigma\left(\mathscr{L}_{\mathrm{A}_{2}, \mathrm{C}_{2}}\right)$ and all associated Jordan blocks of $\lambda_{i}$ are $1 \times 1$. In fact, by Lemma 12, we know that $\sigma\left(\mathscr{L}_{A_{0}, C}\right) \subset C^{-}$. By Theorem 5, $\sigma\left(A_{0}\right) \subset C^{-}$, so $\sigma\left(A_{2}\right) \subset$ $C^{-}$. Finally,

$$
\begin{aligned}
& S^{\prime}\left(A_{0}+B R^{-1} B^{\prime} H\right) S \\
& \quad=\left(\begin{array}{cc}
A_{1} & * \\
0 & -W^{-1} A_{2}^{\prime} W-W^{-1} C_{2}^{\prime} W C_{2}
\end{array}\right) .
\end{aligned}
$$

Since $\left|\operatorname{Re}\left(\lambda_{i}\right)\right|>\left|\operatorname{Re}\left(\mu_{i} \mu_{j}\right)\right|$ for $\lambda_{i} \in \sigma\left(A_{2}\right)$ and $\mu_{i}, \mu_{j} \in$ $\sigma\left(C_{2}\right)$, there exists a $\theta_{i} \in \sigma\left(-W^{-1} A_{2} W-W^{-1} C_{2}^{\prime} W C_{2}\right)$ such that $\operatorname{Re}\left(\theta_{i}\right)>0 . \sigma\left(-W^{-1} A_{2} W-W^{-1} C_{2}^{\prime} W C_{2}\right) \subset \sigma\left(A_{0}+\right.$ $\left.B R^{-1} B^{\prime} H\right)=\sigma\left(A_{0}+B R^{-1} B^{\prime}(\widetilde{P}-\widehat{P})\right)=\sigma\left(A-B R^{-1} B^{\prime} \widetilde{P}+\right.$ $\left.B R^{-1} B^{\prime}(\widetilde{P}-\widehat{P})\right)=\sigma\left(A-B R^{-1} B^{\prime} \widehat{P}\right)$. It directly provides $\sigma\left(\mathscr{L}_{K}\right)$ of system (8) must have unstable modes by Theorem 4 where $K=-R^{-1} B^{\prime} \widehat{P}$; namely, there is a $\lambda \in \sigma\left(\mathscr{L}_{K}\right)$ such that $\lambda>0$. It contradicts with $\sigma\left(\mathscr{L}_{K}\right)$ of system (8) which is contained in $C^{-0}$. Hence $H=0$. That is, $\widetilde{P}=\widehat{P}$. The proof of Theorem 13 is complete.

Remark 14. In fact, the condition $\left|\operatorname{Re}\left(\lambda_{i}\right)\right|>\left|\operatorname{Re}\left(\mu_{i} \mu_{j}\right)\right|$ for $\lambda_{i} \in \sigma\left(A-B R^{-1} B^{\prime} \widetilde{P}\right)$ and $\mu_{i}, \mu_{j} \in \sigma(C)$ is a little stronger. From the proof of Theorem 13, if there exists a $\theta_{i} \epsilon$ $\sigma\left(-W^{-1} A_{2} W-W^{-1} C_{2}^{\prime} W C_{2}\right)$ such that $\operatorname{Re}\left(\theta_{i}\right)>0$, the result still holds.

Remark 15. In fact, Theorem 13 gives a positive proof of Conjecture 10 when $D=0$ in a large extent. That is, the result of Conjecture 10 holds for a big class of stochastic systems.

Corollary 16. Assume system $(A, B, C)$ is mean square stabilizable; if GARE (9) admits a strong solution $\widehat{P}$ such that $\sigma\left(\mathscr{L}_{A-B R^{-1} B^{\prime} P, C}\right) \subset C^{-}$, then it is also the maximal solution.

Proof. If GARE (9) admits a strong solution $\widehat{P}$ such that $\sigma\left(L_{A_{0}, C}\right) \subset C^{-}$; that is, $\lambda_{i} \in \sigma\left(\mathscr{L}_{A-B R^{-1} B^{\prime} \widehat{P}, C}\right) \neq 0$, then $\widehat{P}$ is a feedback stabilizing solution. Hence it is also the maximal solution by the uniqueness of stabilizing solution.

Remark 17. Under the conditions of Theorem 13, either $\sigma\left(\mathscr{L}_{A-B R^{-1} B^{\prime} P, C}\right) \subset C-C^{-0}$ or $\sigma\left(\mathscr{L}_{A-B R^{-1} B^{\prime} P, C}\right) \subset C^{-}$holds, where $P$ is the solution of GARE (9).

Now we consider the positive semidefiniteness of the strong solution $P$ of GARE (9); first of all, we give the following condition.

The condition $H_{1}$ : for each $\varepsilon>0$, there exists a $\delta>0$, such that $E\left\|x\left(t, x_{0}\right)\right\|^{2}<\varepsilon$ whenever $t \geq 0$ and $\left\|x_{0}\right\|<\delta$.

Theorem 18. Assume system $(A, B, C)$ is mean square stabilizable. If the strong solution $P$ of GARE (9) has the property that any eigenvalue $\lambda \in \sigma\left(\mathscr{L}_{A-B R^{-1} B^{\prime} P, C}\right)$ satisfying $\operatorname{Re}(\lambda)=0$ is a simple characteristic root, then $P \geq 0$.
Proof. Consider the following three optimal performance values with the constraint of system (8):

$$
\begin{gathered}
V_{1}\left(x_{0}\right)=\inf _{u \in U_{a d}^{\infty}}\left\{E \int_{0}^{+\infty}\left(x^{\prime} Q x+u^{\prime} R u\right) d t,\right. \\
\left.\lim _{t \rightarrow+\infty} E\|x\|=0\right\}, \\
V_{2}\left(x_{0}\right)=\inf _{u \in U_{a d}^{\infty}}\left\{E \int_{0}^{+\infty}\left(x^{\prime} Q x+u^{\prime} R u\right) d t,\right. \\
\left.(8) \text { satisfies } H_{1}\right\}, \\
V_{3}\left(x_{0}\right)=\inf _{u \in U_{a d}^{\infty}}\left\{E \int_{0}^{+\infty}\left(x^{\prime} Q x+u^{\prime} R u\right) d t,\right. \\
\left.x(0)=x_{0}\right\} .
\end{gathered}
$$

Here $U_{a d}^{\infty}$ denotes all square integrable, $\mathscr{F}_{t}$-measurable processes.

In fact, Let $X=E x x^{\prime}$, using Itô formula and the knowledge of Kronecker multiplication in theory of matrices, the stochastic system (4) becomes a deterministic systems in [10]. By Theorem 5.2.3 in [15], the condition $H_{1}$ is equivalent to the eigenvalue, $\lambda \in \sigma\left(\mathscr{L}_{K}\right)$ satisfying $\operatorname{Re}(\lambda)=0$, is a simple characteristic root.

From Lemma 4.1 in [9] and Theorem 5 in [10], we can, respectively, get

$$
V_{1}\left(x_{0}\right)=x_{0} P_{\max } x_{0}, \quad V_{3}\left(x_{0}\right)=x_{0} P_{\min } x_{0},
$$

where $P_{\min }$ and $P_{\max }$ are the minimal and the maximal positive semidefinite solution of GARE (9). Similarly to proof of Theorem 5 in [10], we get $V_{2}\left(x_{0}\right)=x_{0} P x_{0}$. So

$$
x_{0} P_{\min } x_{0} \leq x_{0} P x_{0} \leq x_{0} P_{\max } x_{0}
$$

Hence,

$$
P_{\min } \leq P \leq P_{\max }, \quad P \geq 0 .
$$

Corollary 19. Assume system $(A, B, C, D)$ is mean square stabilizable. If the strong solution $P$ of GARE (5) has the property that any eigenvalue

$$
\lambda \in \sigma\left(\mathscr{L}_{A-B\left(R+D^{\prime} P D\right)^{-1} B^{\prime}\left(B^{\prime} P+D^{\prime} P C\right), C-D\left(R+D^{\prime} P D\right)^{-1} B^{\prime}\left(B^{\prime} P+D^{\prime} P C\right)}\right)
$$

satisfying $\operatorname{Re}(\lambda)=0$ is a simple characteristic root, then $P \geq 0$. In particular, we consider the case of one dimension,

Theorem 20. Assume system $(A, B, C)$ is mean square stabilizable. If GARE (9) admits a strong solution $P$, then $P$ is also the maximal solution.

Proof. The solutions of GARE (9) are $P=\left(2 A+C^{2} \pm\right.$ $\left.\sqrt{\left(2 A+C^{2}\right)^{2}+4 Q B^{2} R^{-1}}\right) / 2 B^{2} R^{-1}$. When $2 A+C^{2} \neq 0$, 
the strong solution or maximal solution $P=\left(2 A+C^{2}+\right.$ $\left.\sqrt{\left(2 A+C^{2}\right)^{2}+4 Q B^{2} R^{-1}}\right) / 2 B^{2} R^{-1}$; when $2 A+C^{2}=0$ and $Q=0$, the strong solution or maximal solution $P=0$; when $2 A+C^{2}=0$ and $Q \neq 0$, the strong solution or maximal solution $P=\sqrt{4 Q B^{2} R^{-1}} / 2 B^{2} R^{-1}$.

Corollary 21. Assume system $(A, B, C)$ is mean square stabilizable. If GARE (9) only has a solution, then it is the maximal solution; if GARE (9) has two different solutions, the maximal solution of GARE (9) is the stabilizing solution.

Remark 22. In fact, by a series of computations, Conjecture 10 in [9] holds for one dimension.

\section{An Example}

In this section, we will exhibit the effectiveness of Theorem 13 by an example.

Example 1. Consider the following stochastic Itô differential system:

$$
\begin{gathered}
d x=(A x+B u) d t+C x d w \\
x(0)=x_{0} \in R^{n}
\end{gathered}
$$

Choose

$$
\begin{array}{cc}
A=\left(\begin{array}{lll}
4 & 2 & 3 \\
0 & 5 & 2 \\
0 & 0 & 6
\end{array}\right), \quad B=\left(\begin{array}{lll}
1 & 0 & 0 \\
0 & 1 & 0 \\
0 & 0 & 1
\end{array}\right), \quad C=\left(\begin{array}{lll}
1 & 2 & 3 \\
0 & 1 & 2 \\
0 & 0 & 1
\end{array}\right), \\
Q=\left(\begin{array}{lll}
1 & 0 & 0 \\
0 & 1 & 0 \\
0 & 0 & 1
\end{array}\right), & R=\left(\begin{array}{lll}
3 & 2 & 1 \\
2 & 3 & 0 \\
1 & 0 & 1
\end{array}\right) .
\end{array}
$$

Using Matlab, solving the maximal solution of GARE (9), that is, solving the optimal solution of the following SDP problem:

$$
\begin{aligned}
& \max \operatorname{Tr}(P) \\
& \text { subject to }\left(\begin{array}{cc}
P A+A^{\prime} P+C^{\prime} P C+Q & P B \\
B^{\prime} P & R
\end{array}\right) \geq 0, \quad P \geq 0,
\end{aligned}
$$

we can get the following optimal solution $P_{0}$; by Theorem 10 in [3], we know that the given $P_{0}$ is the maximal solution of GARE (9).

One has

$$
P_{0}=\left(\begin{array}{ccc}
7.7145 & 2.7249 & 1.8685 \\
2.7249 & 12.5383 & 5.6915 \\
1.8685 & 5.6915 & 21.4621
\end{array}\right) .
$$

So

$$
\begin{aligned}
A_{0} & =A-B R^{-1} B^{\prime} P_{0} \\
& =\left(\begin{array}{ccc}
-2.0441 & 19.9882 & 37.0819 \\
3.1211 & -10.5049 & -23.2851 \\
4.1756 & -22.6797 & -50.5440
\end{array}\right) .
\end{aligned}
$$

Hence,

$$
\sigma\left(A_{0}\right)=\{-64.4336,2.5783,-1.2377\}, \quad \sigma(C)=\{1,1,1\} .
$$

Obviously, $\left|\operatorname{Re}\left(\lambda_{i}\right)\right|>\left|\operatorname{Re}\left(\mu_{i} \mu_{j}\right)\right|$ for $\lambda_{i} \in \sigma\left(A-B R^{-1} B^{\prime} P_{0}\right)$ and $\mu_{i}, \mu_{j} \in \sigma(C)$. Hence, by Theorem 13, the maximal solution of GARE (9) is only a strong solution.

\section{Conclusion}

In this paper, with the aid of the operator spectrum and generalized Lyapunov equation approach, we prove that the strong solution of GARE is also the maximal solution under certain condition, and it positively proves Conjecture 10 in [9]. From the proof of Theorem 13, we know that the uniqueness of strong solution of GARE (9) has a lot to do with $\sigma(C)$ and the maximal solution $P$. The condition $\left|\operatorname{Re}\left(\lambda_{i}\right)\right|>$ $\left|\operatorname{Re}\left(\mu_{i} \mu_{j}\right)\right|$ for $\lambda_{i} \in \sigma\left(A_{0}\right)$ and $\mu_{i}, \mu_{j} \in \sigma(C)$ ensures that there exists a $\theta_{i} \in \sigma\left(-W^{-1} A_{2} W-W^{-1} C_{2}^{\prime} W C_{2}\right), \operatorname{Re}\left(\theta_{i}\right)>0$. However, whether there exists a weaker condition is still a challenge. Although we don't completely prove Conjecture 10 in [9], we find that the result holds for a big class of stochastic systems; that is, GARE (9) has only a solution $P$ which stabilized system (8) and mean square stabilized system (8); others cannot stabilize system (8). We will look deeper into the problem, which is perhaps related with the time invariant version of the stochastic system and of the generalized Riccati equations in [16].

\section{Conflict of Interests}

The authors declare that there is no conflict of interests regarding the publication of this paper.

\section{Acknowledgments}

This work was supported by the National Natural Science Foundation of China under Grants nos. 61174078 and 61201430, the Research Fund for the Taishan Scholar Project of Shandong Province of China, and SDUST Research Fund (no. 2011KYTD105).

\section{References}

[1] R. Z. Has'minskii, Stochastic Stability of Differential Equations, Sijthoff and Noordhoff, Alphen, The Netherlands, 1980.

[2] H. J. Kushner, Stochastic Stability and Control, Academic Press, New York, NY, USA, 1967.

[3] M. A. Rami and X. Y. Zhou, "Linear matrix inequalities, Riccati equations, and indefinite stochastic linear quadratic controls," IEEE Transactions on Automatic Control, vol. 45, no. 6, pp. 1131$1143,2000$.

[4] T. Damm, Rational Matrix Equations in Stochastic Control, vol. 287 of Lecture Notes in Control and Information Sciences, Springer, New York, NY, USA, 2004.

[5] X. Mao, Stochastic Differential Equations and Their Applications, Horwood, Chichester, UK, 1997. 
[6] Y. Liu, Backward stochastic differential equation and stochastic control system [Ph.D. thesis], Shandong University, Jinan, China, 1999.

[7] S. Boyd, L. El Ghaoui, E. Feron, and V. Balakrishnan, Linear Matrix Inequalities in System and Control Theory, SIAM, Philadelphia, Pa, USA, 1994.

[8] M. D. Fragoso, O. L. V. Costa, and C. E. de Souza, "A new approach to linearly perturbed Riccati equations arising in stochastic control," Applied Mathematics and Optimization, vol. 37, no. 1, pp. 99-126, 1998.

[9] W. H. Zhang, H. S. Zhang, and B.-S. Chen, "Generalized Lyapunov equation approach to state-dependent stochastic stabilization/detectability criterion," IEEE Transactions on Automatic Control, vol. 53, no. 7, pp. 1630-1642, 2008.

[10] W. H. Zhang and B.-S. Chen, "On stabilizability and exact observability of stochastic systems with their applications," Automatica, vol. 40, no. 1, pp. 87-94, 2004.

[11] W. Zhang and B.-S. Chen, "Some properties of generalized Lyapunov equations," in Proceedings of the Chinese Control and Decision Conference (CCDC '11), pp. 3137-3141, Mianyang, China, May 2011.

[12] S. B. Nadler, Continuum Theory: An Introduction, Marcel Dekker, New York, NY, USA, 1992.

[13] A. G. Mazko, "The Lyapunov matrix equation for a certain class of regions bounded by algebraic curves," Soviet Automatic Control, vol. 42, pp. 12-17, 1980.

[14] W. M. Wonham, "On a matrix Riccati equation of stochastic control," SIAM Journal on Control and Optimization, vol. 6, pp. 681-697, 1968.

[15] J. M. Ortega, Matrix Theory, Plenum Press, New York, NY, USA, 1987.

[16] V. M. Ungureanu and V. Dragan, "Nonlinear differential equations of Riccati type on ordered Banach spaces," Electronic Journal of Qualitative Theory of Differential Equations, no. 17, pp. 1-22, 2012. 


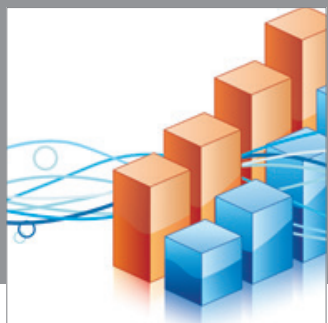

Advances in

Operations Research

mansans

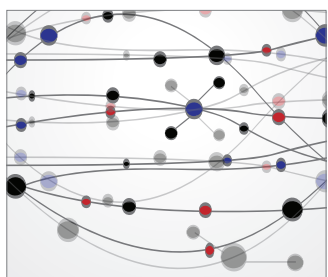

The Scientific World Journal
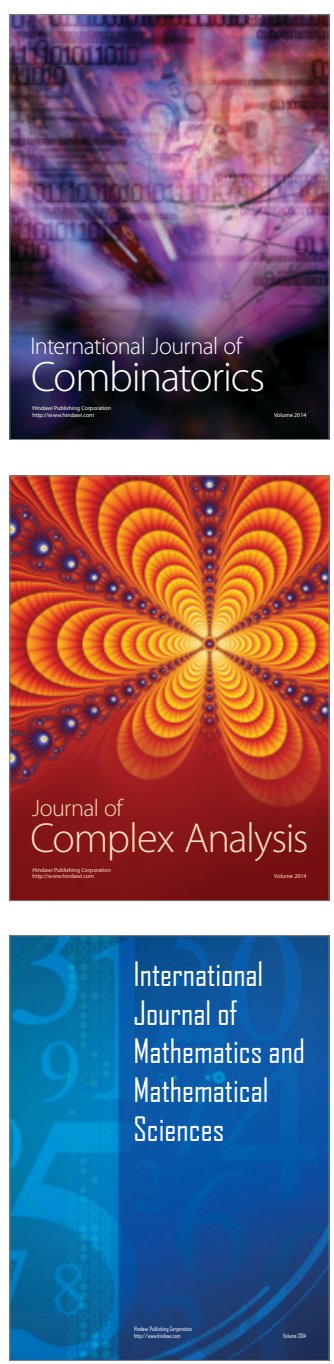
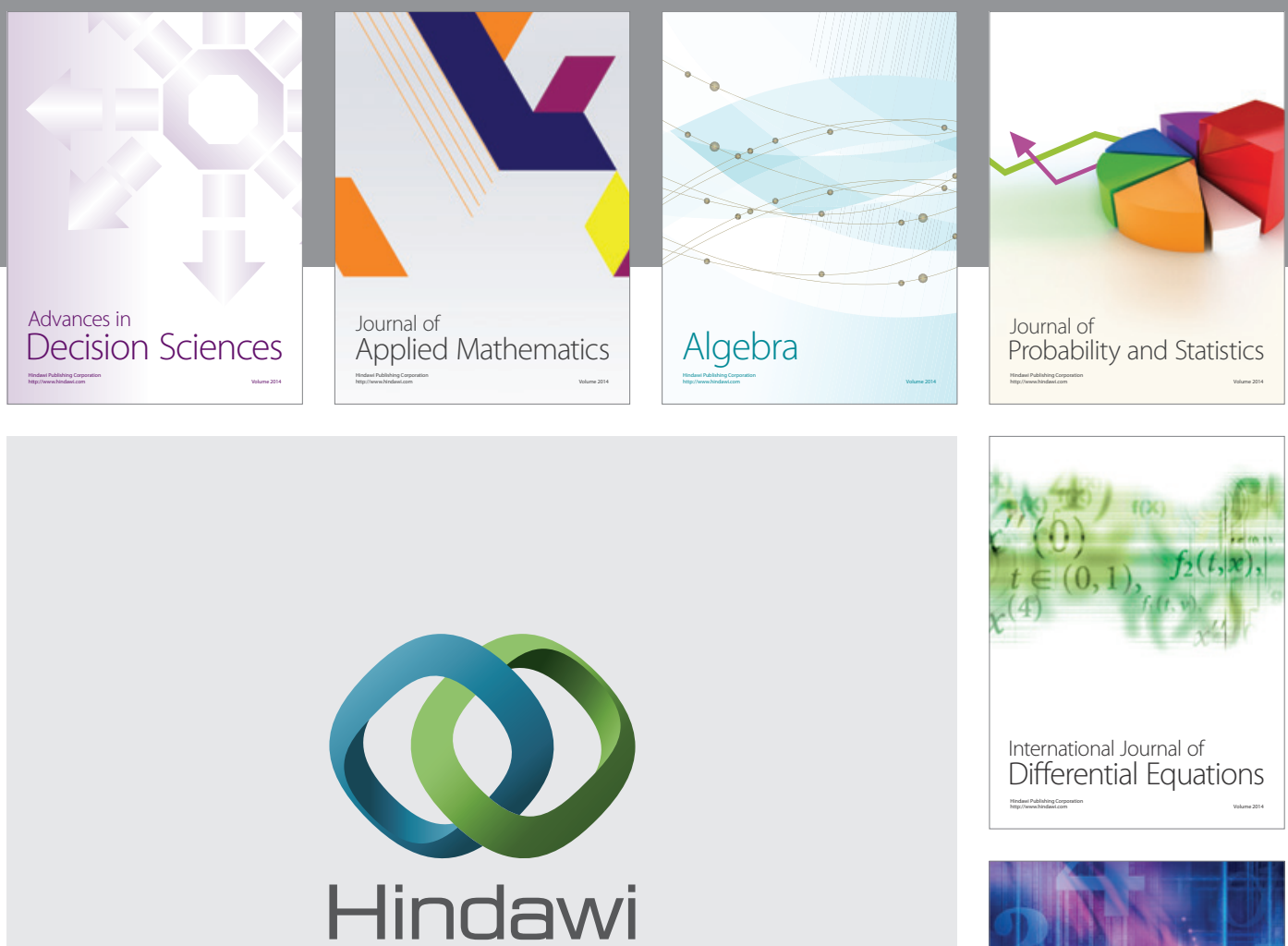

Submit your manuscripts at http://www.hindawi.com
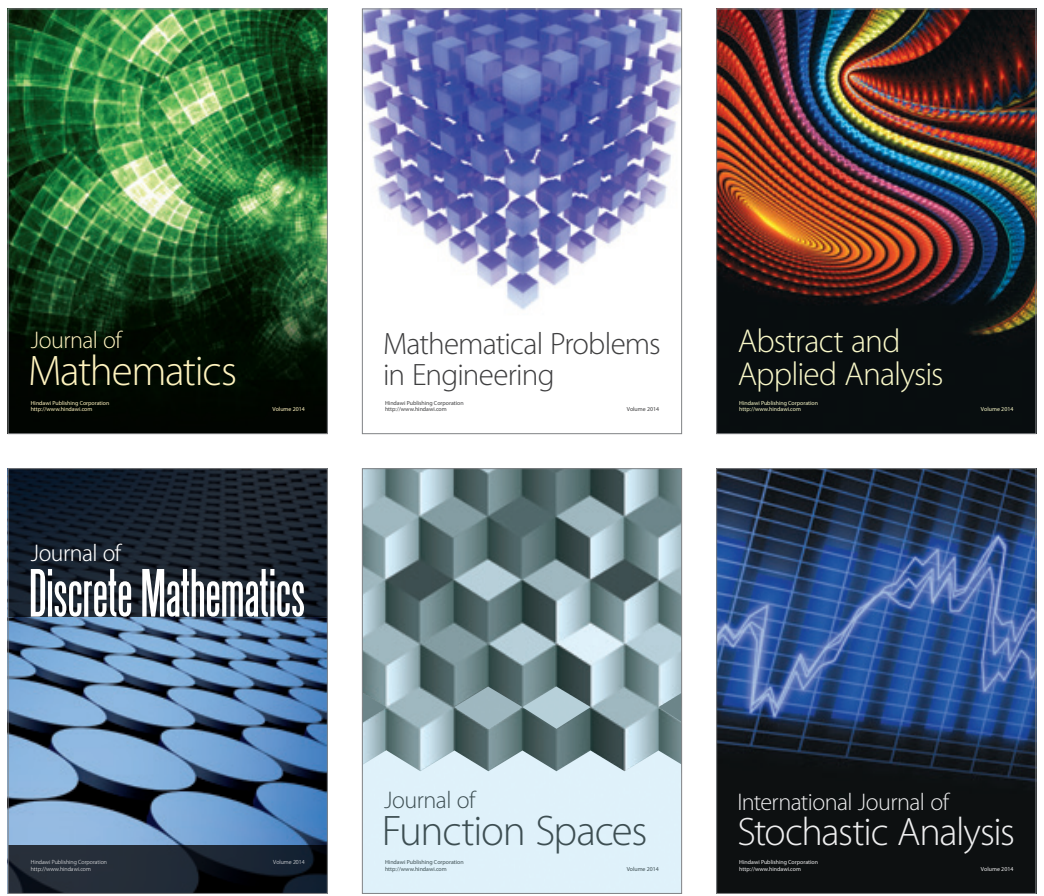

Journal of

Function Spaces

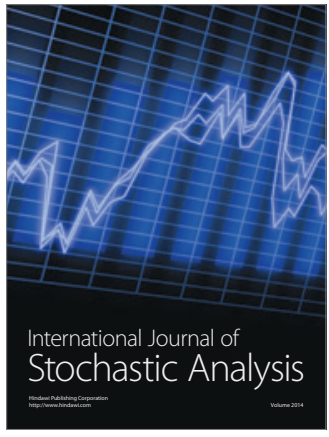

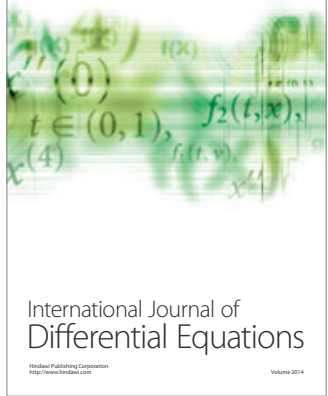
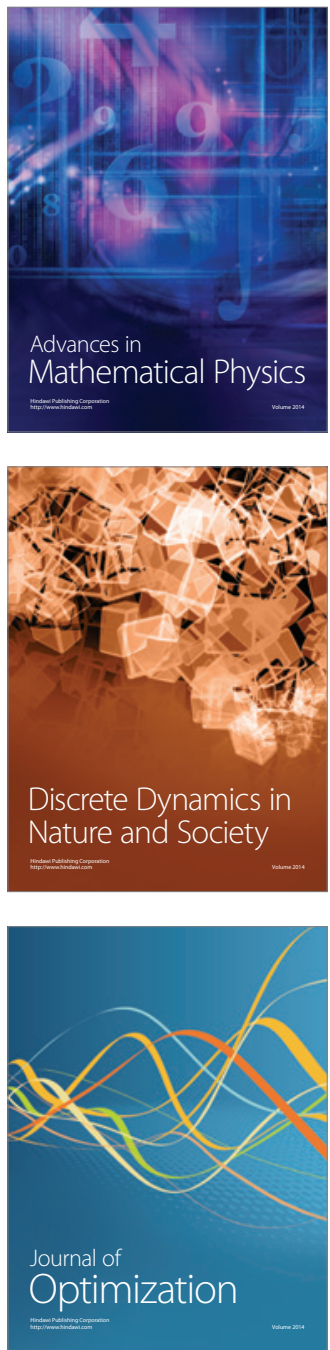